\title{
PARTIAL UPDATE EVEN MIRROR FOURIER NON-LINEAR FILTERS FOR ACTIVE NOISE CONTROL
}

\author{
Vinal Patel, Nithin V. George \\ Department of Electrical Engineering, Indian Institute of Technology Gandhinagar, \\ Gujarat - 382424, India. Email: \{vinal.patel, nithin\}@iitgn.ac.in
}

\begin{abstract}
A non-linear active noise control (ANC) scheme, which is based on an even mirror Fourier non-linear filter has been developed in this paper. A new weight update mechanism for the proposed scheme has been suggested and the range of the learning rate which ensures stability has been derived. The noise mitigation achieved using the new scheme has been compared with that obtained using a functional link artificial neural network (FLANN) based ANC system as well as using a generalized FLANN (GFLANN) based ANC mechanism. The computational complexity of the proposed algorithm has been further reduced by using the concept of partial update signal processing. A simulation study has been carried out to evaluate the effectiveness of the new method. Improved noise reduction at reduced computational load has been provided by the new partial update ANC scheme proposed.
\end{abstract}

Index Terms - Active noise control, FLANN, GFLANN, even mirror filter

\section{INTRODUCTION}

Active noise control (ANC) has recently emerged as an effective method for noise mitigation in the low frequency region of the spectrum. Advances in technology has made it possible to incorporate ANC in devices like headphones [1] and hearing aids [2]. In a basic feed-forward ANC system, a microphone is used to measure the noise to be cancelled and a loudspeaker is employed to produce the necessary anti noise sound. The loudspeaker is controlled by an adaptive controller and an error microphone is used to measure the level of noise control obtained. One of the most popular controllers used in an ANC scheme is a finite impulse response (FIR) filter, the weights of which are updated using a filtered-x least mean square (FxLMS) algorithm [3].

FxLMS algorithm based ANC systems are not efficient when non-linearities are present in the ANC system. This calls for the introduction of non-linear ANC mechanisms, which employ a non-linear filter (trained using a suitable

This work was supported by the Department of Science and Technology, Government of India under the INSPIRE Faculty Award Scheme (IFA-13 ENG-45). learning rule) as the adaptive controller. An adaptive Volterra filter, trained using a Volterra FxLMS (VFxLMS) algorithm has been proposed in [4]. Das and Panda has developed a functional link artificial neural network (FLANN) based ANC system trained using a filtered-s least mean square (FsLMS) algorithm [5]. Several attempts have been made to improve the convergence characteristics of FLANN based ANC systems [6,7]. A generalized FLANN (GFLANN), which incorporates cross terms into FLANN has been recently applied for ANC in [8]. The weights of a GFLANN are updated using a generalized FsLMS (GFsLMS) algorithm.

Carini and Sicuranza has recently proposed an even mirror Fourier non-linear (EMFN) filter for function approximation [9]. The authors have shown improved effectiveness offered by EMFN filter in the function approximation task in comparison with FLANN and GFLANN. In an endeavour to enhance the noise cancellation using FLANN and GFLANN based ANC schemes, we design an EMFN based non-linear ANC system in this paper. A new update rule for the filter is developed and an attempt is made to further reduce the computational complexity by using the principles of partial updates [10].

The rest of the paper is organized as follows: An EMFN based ANC scheme is designed and the corresponding update rule is developed in Section 2. The partial update version of the newly developed update rule is outlined in Section 3. The effectiveness of the proposed scheme in noise mitigation is tested using a simulation study in Section 4 and the concluding remarks are made in Section 5.

\section{EMFN BASED ANC SYSTEM}

A basic feed-forward ANC system consists of a reference microphone which measures the sound to be cancelled, a loudspeaker which produces the necessary anti-noise and an error microphone which senses the sound remaining after cancellation. The sound produced by the loudspeaker is governed by an adaptive controller, with transfer function $W(z)$. Considering $x(n)$ as the primary noise to be cancelled, the tap delayed input signal vector of the EMFN based controller is given by $\boldsymbol{u}(n)=[x(n), x(n-1), \cdots, x(n-L+1)]^{\mathrm{T}}$, where 
$L$ is the tap length. The tap delayed input signal is expanded into $N=L(L+3) / 2$ terms in a second order EMFN. The expanded signal vector is given by

$$
\begin{gathered}
\boldsymbol{X}(n)=\left\{\sin \left[\frac{\pi}{2} x(n)\right], \sin \left[\frac{\pi}{2} x(n-1)\right], \cdots,\right. \\
\sin \left[\frac{\pi}{2} x(n-L+1)\right], \cos [\pi x(n)], \cdots, \cos [\pi x(n-L+1)] \\
\sin \left[\frac{\pi}{2} x(n)\right] \cdot \sin \left[\frac{\pi}{2} x(n-1)\right], \cdots, \sin \left[\frac{\pi}{2} x(n-L+1)\right. \\
. \sin \left[\frac{\pi}{2} x(n-L+1)\right], \sin \left[\frac{\pi}{2} x(n)\right] \cdot \sin \left[\frac{\pi}{2} x(n-2)\right], \cdots, \\
\sin \left[\frac{\pi}{2} x(n-L+3) \cdot \sin \left[\frac{\pi}{2} x(n-L+1)\right], \cdots,\right. \\
\sin \left[\frac{\pi}{2} x(n) \cdot \sin \left[\frac{\pi}{2} x(n-L+1)\right]\right\}^{\mathrm{T}} .
\end{gathered}
$$

and the output of the EMFN is given by

$$
y(n)=\boldsymbol{w}^{T}(n) \boldsymbol{X}(n),
$$

where $\boldsymbol{w}(n)=\left[w_{0}, w_{1}, \ldots, w_{N-1}\right]^{\mathrm{T}}$ is the adaptive weight vector, which needs to be updated using a suitable learning rule. Fig. 1 shows the schematic diagram of an EMFN for order $R=2$. The residual noise $e(n)$ is given by

$$
e(n)=d(n)-y(n) * s(n)
$$

where $d(n)$ is the output of the primary path with transfer function $P(z), s(n)$ is the impulse response of the secondary path (electro-acoustic path from the output of the controller to the output of the error microphone) and $*$ denotes linear convolution operator. The weights of the controller are updated using a gradient descent approach, which minimizes the cost function $\xi=E\left[e^{2}(n)\right]$, where $E[\cdot]$ is the expectation operator. Considering $\xi=E\left[e^{2}(n)\right] \approx e^{2}(n)$, the weights are updated as

$$
\begin{aligned}
\boldsymbol{w}(n+1) & =\boldsymbol{w}(n)-\frac{\mu}{2} \frac{\partial \xi}{\partial \boldsymbol{w}(n)} \\
& =\boldsymbol{w}(n)+\mu e(n) \boldsymbol{X}^{\prime}(n)
\end{aligned}
$$

which is hereafter referred to as the filtered-m least mean square (FmLMS) algorithm. In (5), $\mu$ is the learning rate and $\boldsymbol{X}^{\prime}(n)=\boldsymbol{X}(n) * s(n)$ is the expanded signal vector filtered through the secondary path. The filtered version of $\boldsymbol{X}(n)$ is obtained by filtering the expanded signal vector through a model of the secondary path, with transfer function $\widehat{S}(z)$.

The range of the learning rate for which the algorithm converges is derived as given below. The Taylor series expansion of the error signal $e(n+1)$ is given by

$$
e(n+1)=e(n)+\frac{\partial e(n)}{\partial \boldsymbol{w}^{T}(n)} \Delta \boldsymbol{w}(n)+\eta
$$

where $\eta$ represents the higher order terms in the Taylor series

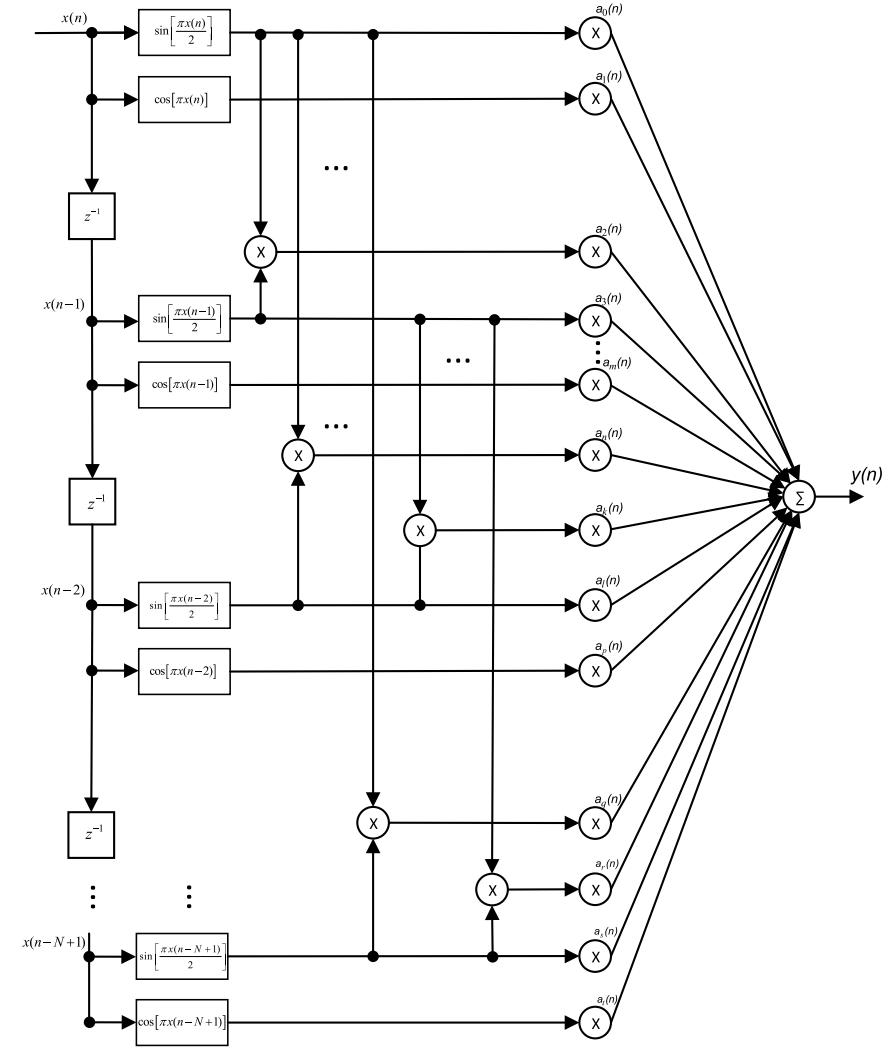

Fig. 1. Schematic diagram of an second order $(R=2)$ EMFN.

expansion and $\Delta \boldsymbol{w}(n)=\boldsymbol{w}(n+1)-\boldsymbol{w}(n)$. From (3) we get

$$
\begin{aligned}
\frac{\partial e(n)}{\partial \boldsymbol{w}^{T}(n)} & =-\boldsymbol{X}^{T}(n) * s(n) \\
& =-\boldsymbol{X}^{\prime T}(n)
\end{aligned}
$$

and

$$
\Delta \boldsymbol{w}(n)=\mu e(n) \boldsymbol{X}^{\prime}(n) .
$$

Substituting (8) and (9) in (6), we get

$$
e(n+1)=\left[1-\mu\left\|\boldsymbol{X}^{\prime}(n)\right\|^{2}\right] e(n) .
$$

In order to ensure convergence, the norm of the terms in the left hand side of (10) should be less than than he norm of the terms in the right hand side of (10), which implies

$$
|e(n+1)| \leq\left|1-\mu\left\|\boldsymbol{X}^{\prime}(n)\right\|^{2} \| e(n)\right| .
$$

In order to satisfy the condition given by (11), we need

$$
\left|1-\mu\left\|\boldsymbol{X}^{\prime}(n)\right\|^{2}\right| \leq 1
$$

which further gives

$$
0<\mu \leq \frac{2}{\left\|\boldsymbol{X}^{\prime}(n)\right\|^{2}}
$$

as the range of $\mu$ for which the proposed FmLMS algorithm converges. 
Table 1. Comparison of computational complexity

\begin{tabular}{|c|c|c|c|c|c|c|c|c|}
\hline & \multicolumn{2}{|c|}{ FsLMS } & \multicolumn{2}{|c|}{ GFsLMS } & \multicolumn{2}{|c|}{ FmLMS } & \multicolumn{2}{|c|}{ PFmLMS } \\
\hline & + & ${ }^{*}$ & + & ${ }^{*}$ & + & $*$ & + & $*$ \\
\hline Controller Output & $N_{f}-1$ & $N_{f}$ & $N_{G}-1$ & $N_{G}$ & $N-1$ & $N$ & $N-1$ & $N$ \\
\hline Filtered Signal & $N_{f}(K-1)$ & $N_{f} K$ & $N_{G}(K-1)$ & $N_{G}^{K}$ & $N(K-1)$ & $N K$ & $N(K-1)$ & $N K$ \\
\hline Weight Update & $N_{f}$ & $N_{f}+1$ & $N_{G}$ & $N_{G}+1$ & $N$ & $N+1$ & $M$ & $M+1$ \\
\hline Total & $N_{f}(K+1)-1$ & $N_{f}(K+2)+1$ & $N_{G}(K+1)-1$ & $N_{G}(K+2)+1$ & $N(K+1)-1$ & $N(K+2)+1$ & $N-K+M-1$ & $N(K+1)+M+1$ \\
\hline
\end{tabular}

\section{PARTIAL UPDATE FMLMS ALGORITHM}

An attempt is made in this section to reduce the computational load offered by the FmLMS algorithm, using the concept of partial update signal processing. In a partial update FmLMS (PFmLMS) algorithm based ANC scheme, only a fraction $\gamma$ of the total weights are updated during every iterations. We have used an $M$-max partial update algorithm, which updates only $M$ of the total $N$ weights in an iteration, based on the weight's contribution to the filter output. The filtered version of the input signal vector may also be written as $\boldsymbol{X}^{\prime}(n)=$ $\left[\alpha_{1}, \alpha_{2}, \ldots, \alpha_{r}, \ldots, \alpha_{N}\right]^{\mathrm{T}}$. The PFmLMS algorithm is given by

$$
\boldsymbol{w}(n+1)=\boldsymbol{w}(n)+\mu e(n) \boldsymbol{\Lambda}(n) \boldsymbol{X}^{\prime}(n)
$$

where

$$
\boldsymbol{\Lambda}(n)=\left[\begin{array}{cccc}
\lambda_{1}(n) & 0 & \cdots & 0 \\
0 & \lambda_{2}(n) & \cdots & 0 \\
\vdots & \vdots & \ddots & \vdots \\
0 & 0 & \cdots & \lambda_{N}(n)
\end{array}\right]
$$

with

$$
\lambda_{r}(n)= \begin{cases}1 & \text { if }\left|\alpha_{r}(n)\right| \in \max _{1 \leq l \leq N}\left(\left|\alpha_{l}(n)\right|, M\right) \\ 0 & \text { otherwise }\end{cases}
$$

The computational complexity of the proposed PFmLMS algorithm is compared with that of FsLMS, GFsLMS and FmLMS algorithms in Table 2, where $N_{f}$ is the number of weights in FLANN, $N_{G}$ is the number of weights in GFLANN and $K$ is the length of the secondary path model. A significant reduction in computational complexity is evident from the comparison. Fig. 2 shows the block diagram of the proposed PFmLMS algorithm based ANC scheme, where $y_{s}(n)$ is the output of the secondary path.

\section{SIMULATION STUDY}

The effectiveness of the proposed non-linear ANC schemes are evaluated in this section through a simulation study. The study has been carried out in an MATLAB environment and the mean square error (MSE) defined by

$$
\xi=10 \log _{10}\left\{\mathrm{E}\left[e^{2}(n)\right]\right\}
$$

has been used as the metric of evaluation. The noise cancellation offered by the FmLMS and PFmLMS algorithms have

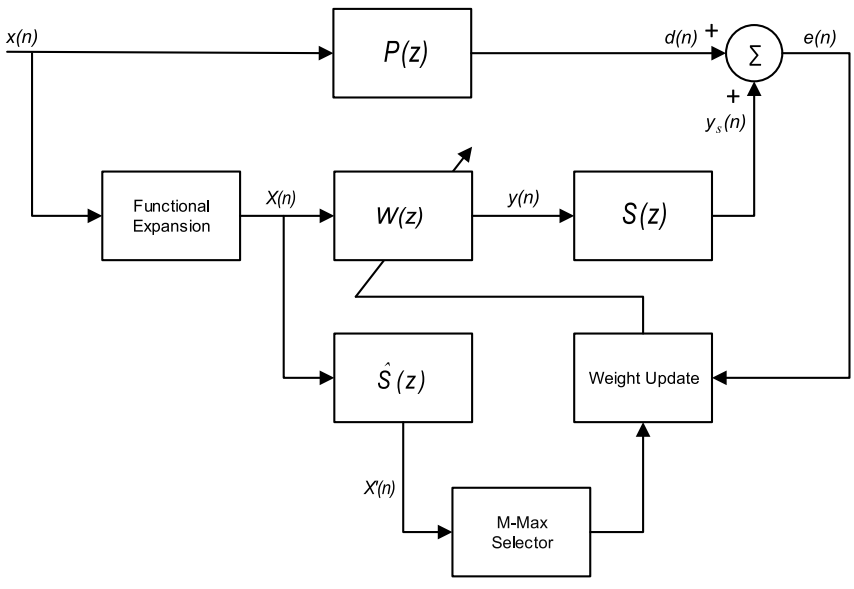

Fig. 2. Schematic diagram of a Partial-update algorithm based ANC system

been compared with that obtained using FsLMS as well as the GFsLMS algorithms. We have assumed perfect modeling of the secondary paths and have considered a measurement noise with a signal to noise ratio of $30 \mathrm{~dB}$. The input signal $x(n)$ employed in all the three experiments given below has been taken as a uniformly distributed random noise in the range $[-0.5,0.5]$ and a tap length of 10 has been employed in all cases. It may be noted that all the learning curves plotted in this study are an average of 200 independent iterations (with a smoothing using a moving average window of length 10) and Table 2 shows the number of coefficients that needs to be updated in every iteration for all the four algorithms compared in the three experiments studied in this paper.

\subsection{Experiment 1}

A set of non-linear primary and secondary paths have been considered in this experiment. The reference signal $x(n)$ and primary noise at the error microphone are related as

$$
\begin{aligned}
d(n)=x & (n)+0.8 x(n-1)+0.3 x(n-2) \\
& +0.4 x(n-3)-0.8 x(n) x(n-1) \\
& +0.9 x(n) x(n-2)+0.7 x(n) x(n-3) .
\end{aligned}
$$

Similarly, the input output relationship of secondary path is defined as

$$
\begin{aligned}
& y_{s}(n)=y(n)+0.35 y(n-1)+0.09 y(n-2) \\
& -0.5 y(n) y(n-1)+0.4 y(n) y(n-2) .
\end{aligned}
$$


Fig. 3 shows the convergence behaviour for all the four filters studied. The MSE computed as an average of last 1000 samples of the experiment are $-20.88,-24.25,-27.38$ and $-27.34 \mathrm{dBs}$ for FsLMS, GFsLMS, FmLMS and PFmLMS $(\gamma=0.7)$ algorithms respectively. The improved noise mitigation capacity of the new schemes are clear from the results obtained. The improvement in noise cancellation has been achieved at a reduced computational load in the case of PFmLMS algorithm. The various other simulation parameters used in this experiment are: FsLMS $\left(R=3, L=10, \mu_{L}=\mu_{N L}=0.05\right)$, GFsLMS $(R=$ $1, N d=2, L=10, \mu_{L}=0.01, \mu_{N L}=0.001$ and $\mu_{C T}=$ 0.005), FmLMS $(R=2, L=10, \mu=0.05)$ and PFmLMS ( $R=2, L=10, \gamma=0.7$ and $\mu=0.05$ ), where $m u_{L}$ and $m u_{N L}$ are the learning rates for the linear and non-linear portions of FLANN and GFLANN.

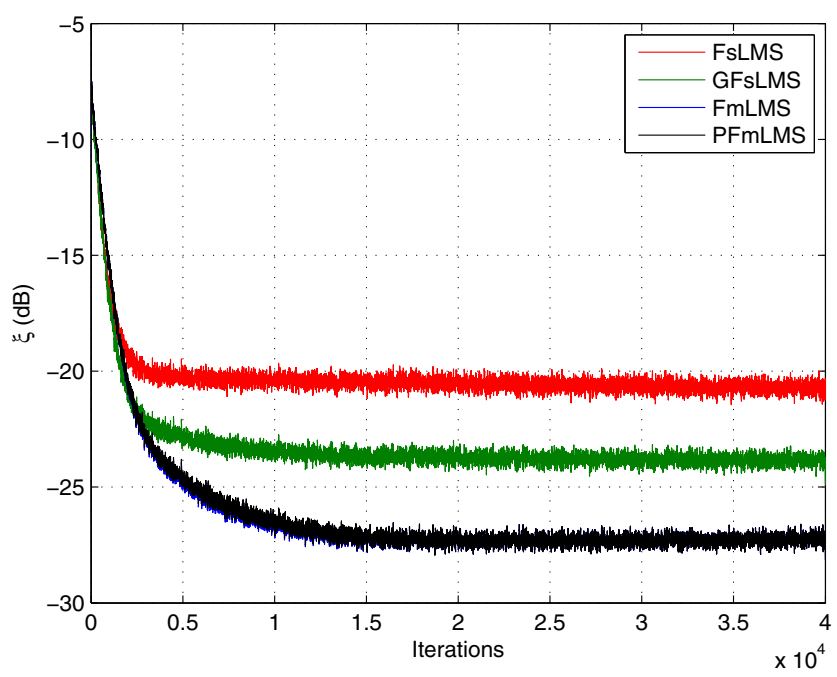

Fig. 3. Experiment 1: Comparison of learning curves for FsLMS, GFsLMS, FmLMS and PFmLMS algorithms.

\subsection{Experiment 2}

In this experiment, we have employed a primary path which is same as that of Experiment 1. The secondary path output is given by

$$
y_{s}(n)=w(n)+0.2 w(n-1)+0.05 w(n-2)
$$

where

$$
w(n)=\tanh [y(n)] .
$$

The average of MSE for the last 1000 samples for the algorithms compared are $-17.83,-22.25,-26.46$ and -26.47 dBs for FsLMS, GFsLMS, FmLMS and PFmLMS $(\gamma=0.7)$ algorithms respectively. Fig. 4 shows the learning curves for the four algorithms and the enhance noise cancellation performance of the proposed algorithms are evident from the results. All other simulation parameters used as same as that of the previous experiment.

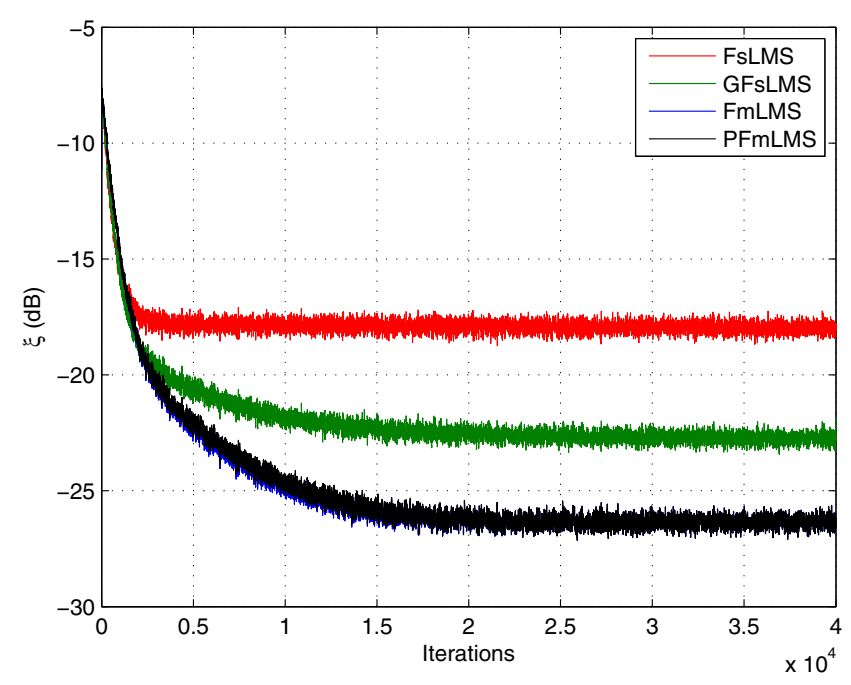

Fig. 4. Experiment 2: Comparison of learning curves for FsLMS, GFsLMS, FmLMS and PFmLMS algorithms.

\subsection{Experiment 3}

The input and output of the primary path used in this experiment are related as

$$
\begin{aligned}
& d(n)=x(n-5)+0.8 x(n-6)+0.3 x(n-7) \\
& \quad+0.4 x(n-2)+0.2 x(n-5) x(n-6) \\
& -0.3 x(n-5) x(n-7)+0.4 x(n-5) x(n-8) .
\end{aligned}
$$

We have used a secondary path transfer function, which is same as that of Experiment 1. The superior noise cancellation offered by the proposed scheme is clear from the comparison of convergence characteristics of the four algorithms shown in Fig. 5. The MSE obtained as an average of the last 1000 samples for the four algorithms compared are -21.38, -25.61, -27.03 and $-27.05 \mathrm{dBs}$ for FsLMS, GFsLMS, FmLMS and PFmLMS $(\gamma=0.7)$ algorithms respectively. The simulation parameters used in this experiment are: FsLMS $(R=3, L=$ $\left.10, \mu_{L}=\mu_{N L}=0.05\right)$, GFsLMS $(R=1, N d=3, L=$ $10, \mu_{L}=0.006, \mu_{N L}=0.001$ and $\left.\mu_{C T}=0.005\right)$, FmLMS ( $R=2, L=10, \mu=0.05)$ and PFmLMS $(R=2, L=$ $10, \gamma=0.7$ and $\mu=0.05$ ). The improvement in noise cancellation and lower computational load offered by PFmLMS algorithm can be seen from the comparisons made in Table 2. The effect of $\gamma$ on computational load as well as MSE can also be observed from the Table. 


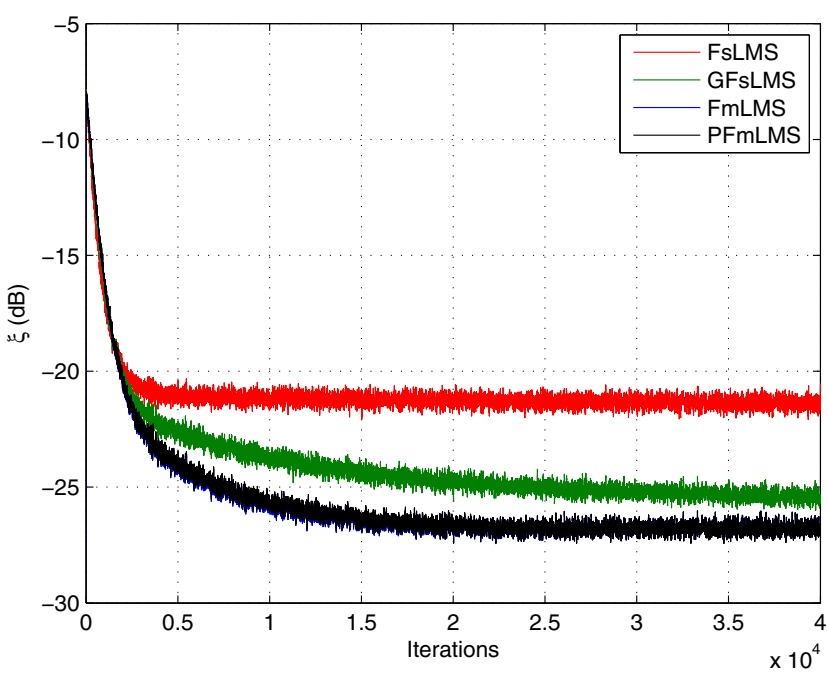

Fig. 5. Experiment 3: Comparison of learning curves for FsLMS, GFsLMS, FmLMS and PFmLMS algorithms.

Table 2. Comparison of computational complexity and MSE for the three experiments

\begin{tabular}{c||c|c|c|c|c|c}
\hline \multicolumn{1}{c||}{} & \multicolumn{3}{c|}{ Number of Coefficients } & \multicolumn{3}{c}{ MSE $(\xi)$} \\
\cline { 2 - 7 } & EX 1 & EX 2 & EX 3 & EX 1 & EX 2 & EX 3 \\
\hline \hline FsLMS & 70 & 70 & 70 & -20.88 & -17.83 & -21.38 \\
GFsLMS & 64 & 64 & 78 & -24.25 & -22.25 & -25.61 \\
FmLMS & 65 & 65 & 65 & -27.38 & -26.46 & -27.03 \\
PFmLMS $(\gamma=0.9)$ & 59 & 59 & 59 & -27.47 & -25.95 & -26.68 \\
PFmLMS $(\gamma=0.8)$ & 52 & 52 & 52 & -27.29 & -26.26 & -26.96 \\
PFmLMS $(\gamma=0.7)$ & 46 & 46 & 46 & -27.34 & -26.47 & -27.05 \\
PFmLMS $(\gamma=0.6)$ & 39 & 39 & 39 & -27.39 & -26.43 & -26.88 \\
PFmLMS $(\gamma=0.5)$ & 33 & 33 & 33 & -27.27 & -26.39 & -26.82 \\
\hline
\end{tabular}

\section{CONCLUSIONS}

A novel non-linear ANC scheme, which is based on even mirror Fourier non-linear filters has been proposed in this paper. A new weight update mechanism (FmLMS algorithm) for the proposed scheme has been suggested and a simulation study has been carried out to evaluate the effectiveness of the new method. The computational complexity of the proposed FmLMS algorithm has been further reduced by designing a partial update FmLMS algorithm. Simulation study has revealed the improved noise mitigation offered by PFmLMS algorithm based ANC system in comparison with other schemes at a reduced computational load.

\section{ACKNOWLEDGEMENTS}

This work was supported by the Department of Science and Technology, Government of India under the Fast Track Scheme for Young Scientists (SERB/ET-0018/2013).

\section{REFERENCES}

[1] S. M Kuo, S. Mitra, and W.S. Gan, "Active noise control system for headphone applications," IEEE Transactions on Control Systems Technology, vol. 14, no. 2, pp. 331-335, 2006.

[2] R. Serizel, M. Moonen, J. Wouters, and S.H. Jensen, "Integrated active noise control and noise reduction in hearing aids," IEEE Transactions on Audio, Speech, and Language Processing, vol. 18, no. 6, pp. 11371146, Aug 2010.

[3] S. M. Kuo and D. R. Morgan, Active Noise Control Systems : Algorithms and DSP Implementations, Wiley, New York, 1996.

[4] L. Z. Tan and Jean Jiang, "Filtered-X second-order Volterra adaptive algorithms," Electronics Letters, vol. 33, no. 8, pp. 671-672, Apr. 1997.

[5] D. P. Das and G. Panda, "Active mitigation of nonlinear noise processes using a novel filtered-s LMS algorithm," IEEE Transactions on Speech and Audio Processing, vol. 12, no. 3, pp. 313-322, May 2004.

[6] E. P. Reddy, D. P. Das, and K. M. M. Prabhu, "Fast exact multichannel FSLMS algorithm for active noise control," Signal Processing, vol. 89, no. 5, pp. 952956, May 2009.

[7] N. V. George and G. Panda, "On the development of adaptive hybrid active noise control system for effective mitigation of nonlinear noise," Signal Processing, vol. 92, no. 2, pp. 509-516, Feb. 2012.

[8] G.L. Sicuranza and A. Carini, "A generalized FLANN filter for nonlinear active noise control," IEEE Transactions on Audio, Speech, and Language Processing, vol. 19, no. 8, pp. 2412-2417, Nov 2011.

[9] A. Carini and G.L. Sicuranza, "Fourier nonlinear filters," Signal Processing, vol. 94, pp. 183-194, 2014.

[10] K. Dogancay, Partial-update adaptive signal processing: Design Analysis and Implementation, 2008. 